\title{
STREPTOCOCCAL AGGLUTININS IN RHEUMATIC FEVER ${ }^{1}$
}

\author{
By EDITH E. NICHOLLS AND WENDELL J. STAINSBY
}

(From the Second (Cornell) Medical Division and the Pathological Laboratories of Belleoue Hospital, the Cornell Clinic, and the Department of Medicine, Cornell University Medical College, New York)

(Received for publication December 12, 1930)

Although considerable evidence has accumulated that rheumatic fever is a streptococcal infection, attempts to demonstrate specific streptococcal agglutinins in the serums of patients with this disease have met with only a moderate degree of success. Tunnicliff (1) performed agglutination reactions with the serums of twelve rheumatic fever patients against strains of Micrococcus rheumaticus and Streptococcus pyogenes, and demonstrated agglutinins in small amounts in seven instances. Swift and Kinsella (2) failed to find any streptococcal agglutinins in the serums of five patients with rheumatic fever against streptococci which they had recovered from their respective blood cultures. Clawson (3) working with strains of Streptococcus viridans isolated from cases of rheumatic fever, found agglutinins in four out of the five serums examined. Small (4) demonstrated agglutinins for the so-called "streptococcus cardioarthritidis" in the serums of ten patients with rheumatic fever.

In a previous paper the writers, in collaboration with Cecil (5), have reported the isolation of streptococci from the blood and joints of a high percentage of patients with rheumatic fever. These strains, with one exception, belonged to the general group of non-hemolytic streptococci. ${ }^{2}$ Cross agglutination and absorption tests performed with immune rabbit serums indicated that while these streptococci

${ }^{1}$ This investigation was supported in part by the Committee for the Encouragement of Medical Research.

2 There is some debate among bacteriologists as to whether the streptococci which we have isolated from cases of rheumatic fever belong to the viridans or indifferent group. For this reason it seems appropriate to use the more general term, "non-hemolytic streptococci." 
in general showed a tendency to fall into a number of biologic groups, one strain, RB5, had antigenic properties in common with the majority of these rheumatic fever streptococci. By reason of this fact, it occurred to us that this strain (RB5) would be particularly appropriate as an antigen in testing for streptococcal agglutinins in the serums of patients with rheumatic fever. The present communication embraces the results obtained in this investigation.

\section{METHODS}

The technic for carrying out the agglutination reactions was identical with that reported in the preceding study of streptococcal agglutinins in the serums of patients with chronic infectious arthritis.

\section{Rheumatic fever}

The patients selected for study presented definite signs of acute rheumatic infection. The majority of the cases were adults with rheumatic polyarthritis but several were children in whom signs of rheumatic infection consisted of fever, leucocytosis, and rheumatic heart disease.

Agglutination reactions were carried out with the serums from 59 patients with rheumatic fever against strain RB5. Of the 59 serums examined 46, or 78 per cent, showed definite agglutination to a titer of $1: 320$ or more; 39 , or 66 per cent, to a titer of $1: 640$ or more; the serums of the remaining 13 patients gave only slight or negative agglutination reactions (table 1 , chart 1 ).

\section{Controls}

Agglutination reactions against strain RB5 were carried out with the serums of 162 normal persons or patients with other diseases (table 1 , chart 1). It was considered advisable to divide the controls into several groups, namely, chronic infectious arthritis, degenerative arthritis, acute tonsillitis and bronchitis, other febrile diseases, other non-febrile diseases and normal individuals. The serums from 6 healthy rabbits were also studied for streptococcal agglutinins.

The serums of 45 patients with chronic infectious arthritis were examined for agglutinins with the streptococcal strain RB5. Of these, 11, or 24.4 per cent, failed to show any agglutination whatever; 30 , 
or 66.7 per cent, agglutinated at very low titers $(1: 20$ to $1: 160)$. Only 4 of the 45 serums produced agglutinations at a titer of 1:320.

The next control group consisted of 16 patients with degenerative (hypertrophic) arthritis. In this group the highest agglutination titer recorded was $1: 160$.

The serums of 8 patients with acute tonsillitis or acute bronchitis were also tested for streptococcal agglutinins. Three gave no agglutination whatever, while the highest recorded was $1: 80$. In view of the frequency with which tonsillitis is associated with rheumatic

TABLE 1

Titers of agglutinations of strain RB5 with patients' serums

\begin{tabular}{|c|c|c|c|c|c|c|c|c|c|c|c|}
\hline \multirow{2}{*}{ Disease } & \multirow{2}{*}{ 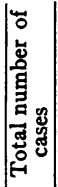 } & \multirow{2}{*}{ 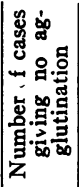 } & \multicolumn{9}{|c|}{ Dilutions of serum $*$} \\
\hline & & & $\stackrel{\text { ণి }}{\underset{-}{*}}$ & $\stackrel{+}{+}$ & 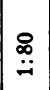 & $\stackrel{8}{\stackrel{8}{ت}}$ & $\stackrel{\text { ్ల }}{-}$ & 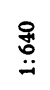 & $\underset{ت}{\stackrel{\Xi}{ت}}$ & $\begin{array}{l}\stackrel{8}{\circ} \\
\stackrel{\leftrightarrow}{*}\end{array}$ & $\underset{\sim}{\stackrel{乛}{3}}$ \\
\hline Rheumatic fever. . & 59 & 1 & 0 . & 1 & 5 & 6 & 7 & 19 & 5 & 10 & 5 \\
\hline Chronic infectious arthritis..... & 45 & 11 & 3 & 9 & 9 & 9 & 4 & 0 & 0 & 0 & 0 \\
\hline Degenerative arthritis......... & 16 & 7 & 3 & 3 & 2 & 1 & 0 & 0 & 0 & 0 & 0 \\
\hline Acute tonsillitis, acute bronchitis. & 8 & 3 & 0 & 4 & 1 & 0 & 0 & 0 & 0 & 0 & 0 \\
\hline Other febrile diseases. . . . . . . . & 23 & 8 & 0 & 8 & 1 & 2 & 1 & 3 & 0 & 0 & 0 \\
\hline Other non-febrile diseases...... & 30 & 10 & 2 & 3 & 6 & 3 & 3 & 2 & 1 & $\mathbf{0}$ & 0 \\
\hline Normal persons......... & 40 & 16 & 6 & 4 & 10 & 4 & 0 & 0 & 0 & $\mathbf{0}$ & 0 \\
\hline Healthy rabbits. .......... & 6 & 4 & 0 & 2 & 0 & 0 & 0 & 0 & 0 & 0 & 0 \\
\hline
\end{tabular}

* The numerals under the various dilutions indicate the number of cases with the limit of agglutination at that dilution.

fever, the results of the agglutination reactions of this group were unexpected.

The next control group consisted of 23 serums from patients with various febrile diseases other than rheumatic fever and subacute bacterial endocarditis, of which only 4 gave agglutination reactions at a titer of $1: 320$ or higher; the remaining 19 gave slight or negative reactions.

In the study of the serums of 30 patients with non-febrile diseases other than arthritis 24 , or 80 per cent, failed to produce any agglutination in dilutions above $1: 160 ; 3$ showed weakly positive reactions, agglutinating to a titer of $1: 320$; one serum from a case of exophthal- 
mic goiter agglutinated strain RB5 to a titer of $1: 640 ; 2$ serums from patients with auricular fibrillation agglutinated to a titer of $1: 1280$ and $1: 640$, respectively.

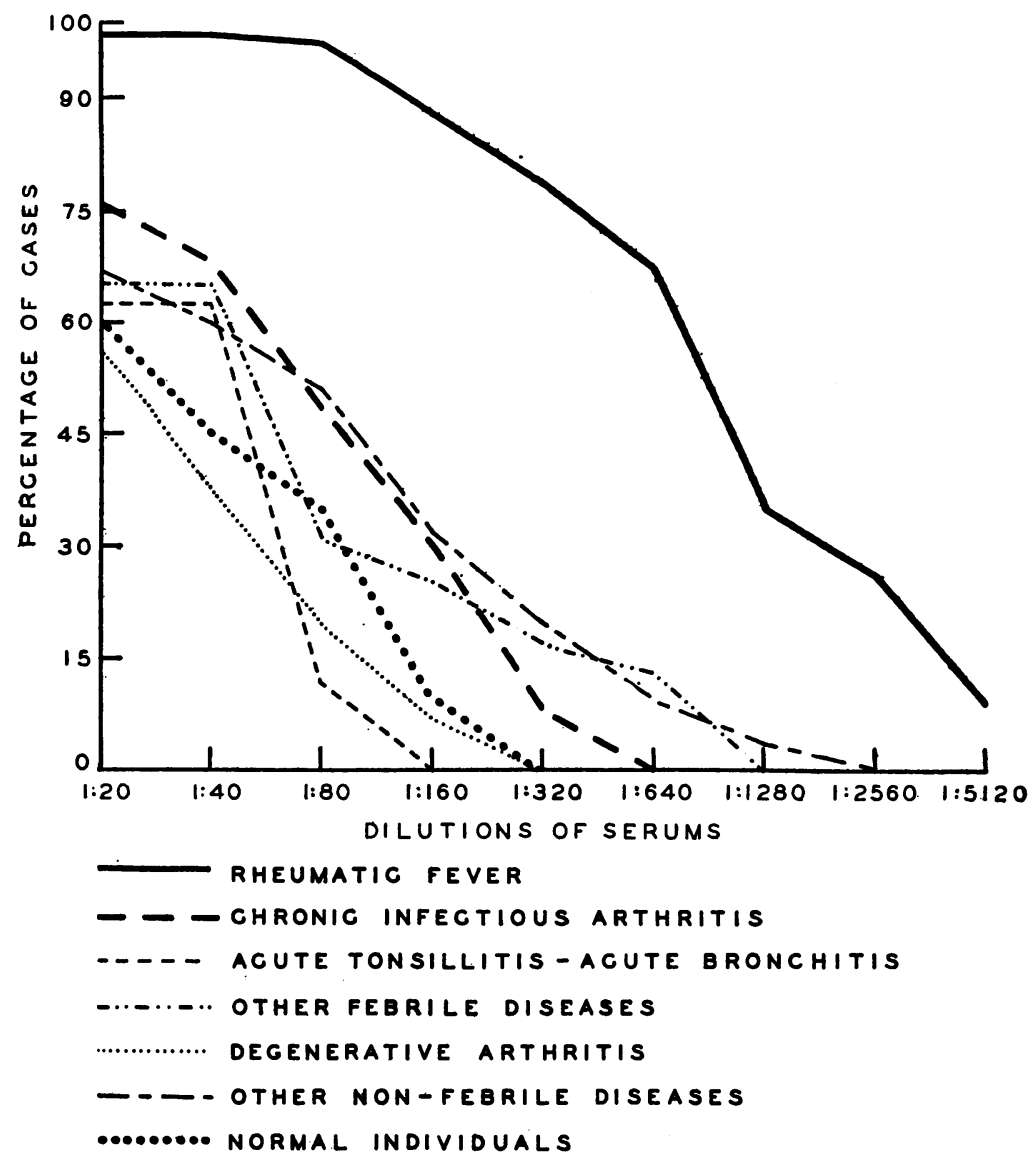

Chart 1. Total Agglutinations with Patients' Serums Against Strain RB5

The serums from 40 normal individuals failed to produce any agglutination of the strain RB5 in dilutions above 1:160.

The highest agglutination with the serums from 6 healthy rabbits against ștrain RB5 was 1:40. 


\section{Chronic polyarthritis following rheumatic fever}

The serums from 3 patients with chronic polyarthritis following rheumatic fever were also studied for streptococcal agglutinins with strain RB5. The titers were 1:640, 1:320, and 1:320, respectively. Control agglutination tests carried out with the same serums against "typical strain" cultures of streptococci from cases of primary chronic infectious arthritis were practically negative.

\section{Subacute bacterial endocarditis}

One of the most interesting phases of this investigation was the study of the serums of 6 patients with subacute bacterial endocarditis.

TABLE 2

Titers of agglutinations with serums of patients with subacute bacterial endocarditis

\begin{tabular}{c|l|c|c}
\hline \multirow{2}{*}{ Patient } & Blood culture & \multicolumn{2}{|c}{ Bacterial antigens } \\
\cline { 3 - 4 } & & RB5* & AB13t \\
\hline 1 & Streptococcus viridans & $1: 320$ & $1: 40$ \\
2 & Streptococcus viridans & $1: 640$ & 0 \\
3 & Streptococcus viridans & $1: 1,280$ & 0 \\
5 & Streptococcus viridans & $1: 5,120$ & 0 \\
6 & Streptococcus viridans & $1: 640$ & 0 \\
& & $1: 1,280$ & $1: 80$ \\
\hline
\end{tabular}
fever.

* RB5 strain of streptococcus recovered from the blood of a patient with rheumatic

† AB13 "typical strain" streptococcus recovered from the blood of a patient with chronic infectious arthritis.

Each of these patients presented the typical clinical picture of the disease, and blood cultures from 5 of the 6 cases yielded streptococcus viridans. In table 2 the agglutination titers of the serums of these 6 patients against strain RB5 and against the "typical strain" of chronic infectious arthritis (AB13) are presented. Each of the serums produced an agglutination to a high titer with the rheumatic fever strain, but showed little or no agglutination with strain AB13.

Diminution of agglutinin content in serums following recovery

In 4 patients with acute rheumatic fever, the agglutinin content of their serums was studied during the convalescent stage of the disease 
as well as during the active period. In each of the 4 cases the second study was made after the patient's temperature had been normal for about one month. The titers of agglutination during the febrile stage were $1: 1280,1: 1280,1: 640$, and $1: 320$, while during the convalescent stage they were $1: 40,1: 160,1: 160$, and $1: 20$, respectively. From these observations it appears that the agglutinins in the serums diminish rapidly following recovery from the disease.

\section{Stability of agglutinins in serums}

In order to determine the stability of the streptococcal agglutinins in the serums of patients with rheumatic fever, agglutination tests with strain RB5 were carried out at weekly intervals on the same

TABLE 3

Titers of agglutinations with joint fluids and corresponding serums from patients with rheumatic fever

\begin{tabular}{c|l|l|l|c}
\hline \multirow{2}{*}{ Patient } & \multicolumn{2}{|c|}{ RB5* antigen $^{*}$} & \multicolumn{2}{c}{ AB13† antigen } \\
\cline { 2 - 3 } & Joint fluid & Serum & Joint fluid & Serum \\
\cline { 1 - 2 } 1 & $1: 20$ & $1: 160$ & 0 & 0 \\
2 & $1: 1,280$ & $1: 2,560$ & 0 & 0 \\
3 & $1: 640$ & $1: 640$ & 0 & 0 \\
4 & $1: 2,560$ & $1: 5,120$ & 0 & $1: 40$ \\
5 & $1: 160$ & $1: 1,280$ & 0 & 0 \\
\hline
\end{tabular}

* RB5 strain of streptococcus recovered from the blood of a patient with rheumatic fever.

† AB13 "typical strain" streptococcus recovered from the blood of a patient with chronic infectious arthritis.

serums. During this period the serums were kept in the refrigerator at almost freezing temperatures. In sharp contrast to the serums from patients with chronic infectious arthritis those from patients with acute rheumatic fever lost their power to agglutinate strain RB5 within 30 days after collection of the specimen.

\section{Streptococcal agglutinins in joint fluids}

Agglutination reactions with strain RB5 were carried out on the joint fluids of 5 patients with rheumatic fever. The technic for testing the joint fluids was similar in every respect to that employed for serums. The agglutinating titers of the joint fluids and of their 
respective serums are presented in table 3 . The agglutination reactions obtained with joint fluids were surprisingly high. Control reactions performed with the typical arthritic strain $\mathrm{AB} 13$ yielded practically no agglutination.

\section{DISCUSSION}

In this study, agglutination reactions with the serums of patients with acute rheumatic fever have been performed against one organism, a non-hemolytic streptococcus, strain RB5, which was originally isolated from the blood of a patient with rheumatic fever. As previously stated, this organism contained antigenic properties in common with the majority of the strains of streptococci recovered in this laboratory from the blood and joints of patients with rheumatic fever. The agglutination tests have shown that the serums, as well as the joint fluids, of most patients with rheumatic fever contain agglutinins for strain RB5 in considerable amounts, while control serums possess little or no agglutinative power for this strain. The results of these agglutination reactions furnish additional evidence that non-hemolytic streptococci are of etiologic importance in rheumatic fever.

The serums of 3 patients with chronic polyarthritis following rheumatic fever produced considerable agglutination of the rheumatic strain RB5, but little or no agglutination of the infectious arthritic strain AB13. These results suggest that the chronic polyarthritis which follows rheumatic fever is not etiologically identical with primary chronic infectious arthritis, and, furthermore, that the former disease is a direct sequel and chronic manifestation of rheumatic fever.

The agglutination reactions of 6 patients with subacute bacterial endocarditis were particularly interesting. The titers of agglutination against strain RB5 were unusually high, and afforded additional evidence of the relationship between rheumatic fever and subacute bacterial endocarditis. One is tempted to speculate as to just how close this relationship is. Are the two diseases identical, but expressions of different phases of a chronic streptococcal infection? Good arguments could be advanced for and against this theory, but such a discussion would be irrelevant in the present article.

In 4 patients with rheumatic fever studied at different stages of the disease, it was found that the agglutinin content of the serums rapidly 
diminished following recovery. In this respect, the agglutinins behave as in other infections such as typhoid fever and pneumonia, where they tend to disappear during or after convalescence.

\section{CONCLUSIONS}

1. Additional evidence is presented in support of the theory that streptococci of the non-hemolytic type are important etiologic agents in rheumatic fever. This evidence consists in the demonstration of streptococcal agglutinins in the serums of rheumatic fever patients.

2. Chronic progressive polyarthritis following rheumatic fever although presenting a clinical picture similar to primary chronic infectious arthritis gives evidence by agglutination reactions of being etiologically different.

3. Further evidence is presented of the etiologic relationship between rheumatic fever and subacute bacterial endocarditis.

\section{BIBLIOGRAPHY}

1. Tunnicliff, Ruth, J. Inf. Dis., 1909, vi, 346. The Opsonic Index in Acute Articular Rheumatism.

2 Swift, H. F., and Kinsella, R. A., Arch. Int. Med., 1917, xix, 381. Bacteriologic Studies in Acute Rheumatic Fever.

3. Clawson, B. J., J. Inf. Dis., 1925, xxxvi, 444. Studies on the Etiology of Acute Rheumatic Fever.

4. Small, J. C., Am. J. Med. Sci., 1928, clxxv, 638. Rheumatic Fever. I. Observations Bearing on the Specificity of Streptococcus Cardioarthritidis in Rheumatic Fever and Sydenham's Chorea.

5. Cecil, R. L., Nicholls, E. E., and Stainsby, W. J., J. Exp. Med., 1929, 1, 617. Bacteriology of the Blood and Joints in Rheumatic Fever. 